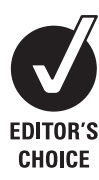

See Editorial, p 97

- Additional details of the techniques, statistical analysis and figures are published online only at http://thorax.bmj.com/ content/vol64/issue2

${ }^{1}$ Department of Radiology, Papworth Hospital NHS

Foundation Trust, Papworth

Everard, Cambridge, UK;

${ }^{2}$ Oxford Centre for Respiratory

Medicine and University of

Oxford, Oxford Radcliffe

Hospital, Oxford, UK;

${ }^{3}$ Department of Radiology,

Oxford Radcliffe Hospital,

Oxford, UK

Correspondence to:

Dr F V Gleeson, Department of

Radiology, Oxford Radcliffe

Hospital, Headington, Oxford

0X3 7LJ, UK; fergus.gleeson@

nds.ox.ac.uk

NRO and NMR are joint first authors with equal roles in design, delivery and publication.

Received 29 June 2008 Accepted 23 September 2008 Published Online First

13 October 2008

\title{
Thoracic ultrasound in the diagnosis of malignant pleural effusion
}

\author{
N R Oureshi, ${ }^{1}$ N M Rahman, ${ }^{2}$ F V Gleeson ${ }^{3}$
}

\begin{abstract}
Background: Malignant pleural effusion (MPE) is a common clinical problem with described investigation pathways. While thoracic ultrasound (TUS) has been shown to be accurate in pleural fluid detection, its use in the diagnosis of malignant pleural disease has not been assessed. A study was undertaken to assess the diagnostic accuracy of TUS in differentiating malignant and benign pleural disease.
\end{abstract}

Methods: 52 consecutive patients with suspected MPE underwent TUS and contrast-enhanced CT (CECT). TUS was used to assess pleural surfaces using previously published CT imaging criteria for malignancy, diaphragmatic thickness/nodularity, effusion size/nature and presence of hepatic metastasis (in right-sided effusions). A TUS diagnosis of malignant or benign disease was made blind to clinical data/other investigations by a second blinded operator using anonymised TUS video clips. The TUS diagnosis was compared with the definitive clinical diagnosis and in addition to the diagnosis found at CECT.

Results: A definitive malignant diagnosis was based on histocytology (30/33; 91\%) and clinical/CT follow-up (3/33; 9\%). Benign diagnoses were based on negative histocytology and follow-up over 12 months in 19/19 patients. TUS correctly diagnosed malignancy in 26/33 patients (sensitivity $73 \%$, specificity $100 \%$, positive predictive value $100 \%$, negative predictive value $79 \%$ ) and benign disease in 19/19. Pleural thickening $>1 \mathrm{~cm}$, pleural nodularity and diaphragmatic thickening $>7 \mathrm{~mm}$ were highly suggestive of malignant disease.

Conclusion: TUS is useful in differentiating malignant from benign pleural disease in patients presenting with suspected MPE and may become an important adjunct in the diagnostic pathway.

Investigation of pleural effusion of unknown aetiology is well described in British, American and European guidelines. ${ }^{1-3}$ These guidelines and other papers ${ }^{4}$ recommend clinical evaluation, basic radiological investigation and diagnostic pleural fluid sampling in the majority of unilateral pleural effusions. Malignancy remains the most common cause of unilateral pleural effusion in the UK and USA, with an estimated 250000 new cases of malignant pleural effusions per year. ${ }^{25}$ Cytologypositive pleural fluid is found in $60 \%$ of cases of malignant pleural effusion, ${ }^{16-8}$ with a substantially lower positive rate in mesothelioma, ${ }^{9}$ and further investigations to establish diagnosis are recommended in the context of cytology-negative unilateral pleural exudates. ${ }^{1-4}{ }^{10}$ Thoracic CT scanning with contrast enhancement (contrast-enhanced CT, CECT) is a sensitive and specific test for malignant pleural disease, ${ }^{11}$ with morphological criteria established in previous studies. ${ }^{12}{ }^{13}$ CECT is recommended as the next investigation, with a view to subsequent histological diagnosis (blind, image-guided or thoracoscopic pleural biopsy). ${ }^{414}$

Thoracic ultrasound (TUS) is a valuable clinical tool which is increasingly being performed by chest physicians. In the UK, guidelines have recently been published with suggested training for physicians with an interest in practising TUS. ${ }^{15}$ Hitherto, the role of TUS has been limited to pleural fluid detection (with high sensitivity) and image-guided techniques (thoracocentesis, drain placement, lung biopsy). ${ }^{14}$

The sonographic appearance of malignant pleural effusion and the value of ultrasound in determining the nature of pleural effusion have been described in previous studies. ${ }^{16}{ }^{17}$ However, there are no published studies to our knowledge which have assessed the diagnostic accuracy of ultrasound for malignancy in patients with suspected but undiagnosed malignant pleural effusion.

The primary aims of this study were therefore (1) to assess the sensitivity and specificity of ultrasound in the detection of malignant disease in patients with suspected malignant pleural effusion using established morphological criteria from CECT; and (2) to investigate the use of other morphological characteristics on TUS associated with malignant pleural disease. In addition, the overall TUS diagnostic rate and CECT diagnostic rate were compared, in comparison to a definitive clinical diagnosis for malignant effusion.

\section{METHODS}

\section{Subjects}

The study was undertaken in a tertiary referral centre for respiratory/pleural disease and involved consecutive patients presenting with unilateral pleural effusion of unknown aetiology from both inpatient and outpatient settings.

\section{Inclusion criteria}

- Chest radiograph evidence of pleural effu$\operatorname{sion}(\mathrm{s})$.

- No established diagnosis (malignant or otherwise) of the cause of pleural effusion.

- The patient would in normal clinical practice undergo further investigations to establish the cause of pleural effusion.

\section{Exclusion criteria}

- A clinical and/or histological diagnosis had been established.

- Clinical and radiographic features of empyema. 
- The patient was too ill to warrant further investigation in normal clinical practise (eg, moribund patients).

Patients were identified by a respiratory trainee (NMR) and referred for TUS with no clinical information on past history, presenting features or relevant investigations.

We routinely perform a TUS in all patients presenting as above before biopsy, drain insertion or thoracoscopy and, as such, this study was considered an audit of our current practice for which local ethics committee approval is not required in our institution.

\section{Ultrasound}

Precise details of the ultrasound technique and operators are given in the online supplement. All patients underwent TUS, before which the most recent chest radiograph was reviewed. TUS was performed without clinical history and previous threedimensional (CT/MRI) imaging data. Anonymised video clips and still images of the examination were generated. From these TUS findings an overall diagnosis of malignant or benign pleural disease was recorded on a reporting proforma (see online supplement). Anonymised TUS data were then reviewed separately by a consultant radiologist experienced in thoracic ultrasound (FVG), blind to clinical history, previous investigations (including radiology), physical status and appearance of the patient. The final results of the blind analysis were recorded by one of the authors (NRQ).

\section{TUS diagnosis}

Morphological criteria established as sensitive and specific to malignant pleural disease on CECT were used as the basis of TUS diagnosis. If a patient had any one of the following criteria on TUS, a diagnosis of malignant disease was recorded:

- Diaphragmatic and parietal pleural nodule or nodules.

- Pleural thickening $>1 \mathrm{~cm}$.

- Hepatic metastasis.

A provisional diagnosis of malignant or benign pleural disease was recorded on the proforma prior to other investigations and separately by each operator.

\section{Contrast-enhanced CT}

Precise details of CECT examinations are presented in the online supplement. CECT examinations were reviewed in all cases and were reported blind to the previous TUS result (as the TUS results were anonymised). In $40 / 52$ patients, CECT was conducted in our institution; in $12 / 52$ patients CECT studies had already been performed at the referring hospital. It was not considered ethical to subject these patients to further radiological examination for the purpose of this study. The hard copy CT images from these institutions were reviewed blind to the TUS result. An overall CT diagnosis of malignant or benign disease was recorded based on the criteria of Leung et $a l,{ }^{12}$ in addition to the presence of metastatic disease or clear intraparenchymal evidence of malignancy (see online supplement).

\section{Definitive diagnosis}

A histocytological diagnosis of malignancy was taken as definitive confirmation of malignant aetiology. Microbiological confirmation (most commonly tuberculosis) was taken as definitive confirmation of benign aetiology. In the absence of the above, the combination of clinical features and/or prolonged radiological follow-up as appropriate was used to define the final diagnosis. Given that around $8 \%$ of patients with apparently benign histology on pleural biopsy develop malignancy over time, ${ }^{18}$ all patients with benign disease were followed up (as part of routine clinical practice in our or the host institution) for a minimum of 12 months to confirm that malignant disease did not develop. For the purposes of this study, "definitive diagnosis" was considered to be the diagnosis imparted to the patient and on which basis the patient was treated (see Results).

\section{Statistical analysis}

Details of the statistical analysis are given in the online supplement.

\section{RESULTS \\ Patients}

From January to September 2005, 52 consecutive patients were recruited. Their baseline characteristics are summarised in table 1.

\section{Definitive diagnosis}

Malignancy

Thirty-three patients of median age 68 years (range 41-89) were diagnosed with malignant disease and 19 patients of median age 68 years (range 22-88) were diagnosed with benign disease (table 1). The mode of final diagnosis is shown in table 2. In 30/ 33 cases $(91 \%)$ the definitive diagnosis of malignancy was based on histocytological confirmation. In the remaining 3 cases (9\%) the diagnosis of pleural malignancy was based on follow-up CT appearances over a period of 6 months in association with a clinical course (including death in all cases) consistent with malignant disease.

\section{Benign disease}

After initial negative histocytological investigations, all cases of benign disease (19/19) were followed up for a minimum period of 12 months. This included patients with positive microbiology or "inflammation" on pleural biopsies. There were no clinical or radiological features of malignancy during the followup period.

\section{TUS overall characteristics}

Pleural effusion was right-sided in 32 patients, left-sided in 13 and bilateral in 7 (in which cases the larger effusion was assessed as this was considered to be the clinically relevant side). The effusions were considered large in 13 patients, moderate in 24 and small in 15 cases (table 3). Effusions were anechoic in 47/ 52 (90\%), 22 were septated and 5 were diffusely echogenic. Liver ultrasound demonstrated metastases in 2 patients and was the sole abnormality suggestive of malignancy in 1 (table 3 ).

\section{TUS diagnoses compared with definitive diagnosis}

There was good inter-observer agreement between the two TUS operators in the overall diagnosis of benign or malignant pleural effusion (kappa 0.96, p<0.001), and the results of the more experienced operator (FVG) have been used for analysis in all subsequent results.

The diagnosis based on the TUS overall correctly diagnosed $26 / 33$ patients with malignant disease and all 19 patients with benign disease (sensitivity $79 \%$ (95\% CI 61\% to 91\%), specificity $100 \%$ (95\% CI $82 \%$ to $100 \%$ ), positive predictive value (PPV) $100 \%$ and negative predictive value (NPV) $73 \%$ for differentiating 
Table 1 Summary of baseline patient characteristics and final diagnoses

\begin{tabular}{ll}
\hline Characteristics & \\
\hline Number of patients & 52 \\
Women & 17 \\
Median (range) age (years) & 68 (22-89) \\
Men & 35 \\
Median (range) age (years) & 61 (41-86) \\
Malignant disease (n=33) & \\
Mesothelioma & $14^{*}$ \\
Adenocarcinoma & 13 \\
Lymphoma & 2 \\
Small cell lung carcinoma & 2 \\
Squamous cell lung carcinoma & 1 \\
Bronchogenic carcinoma (radiological diagnosis only) & 1 \\
Benign disease (n = 19) & \\
Congestive cardiac failure & 6 \\
Benign pleural fibrosis & 7 \\
Parapneumonic effusion & 2 \\
Tuberculous pleuritis & 2 \\
Benign asbestos-related effusion & 1 \\
Constrictive pericarditis & 1 \\
\hline *12 cases of mesothelioma were diagnosed histocytologically; the \\
remaining 2 were diagnosed on clinical and radiological follow-up \\
with a history of asbestos exposure. \\
$\quad$
\end{tabular}

malignant from benign pleural disease with a likelihood ratio $(\mathrm{LR}+\mathrm{ve})=$ infinite $)$.

Parietal pleural thickening was detected in 21 patients, measuring $>1 \mathrm{~cm}$ in $14 / 33(42 \%)$ patients with a malignant effusion and $1 / 19(5 \%)$ patients with a benign effusion $\left(\chi^{2} 1 \mathrm{df}\right.$ 8.11, $\mathrm{p}=0.004)$, and $<1 \mathrm{~cm}$ in $2 / 33(6 \%)$ malignant and $4 / 19$ $(21 \%)$ benign patients $\left(\chi^{2} 1 \mathrm{df} 2.66, p=0.10\right)$. Using a TUS threshold value of pleural thickening $>1 \mathrm{~cm}$ as suggestive of malignancy, TUS has a sensitivity of $42 \%$ (95\% CI $26 \%$ to $61 \%$ ), specificity $95 \%$ (95\% CI $74 \%$ to $99 \%$ ), PPV $93 \%$ and NPV $49 \%$ for differentiating malignant from benign disease (LR+ve $=8.4$; see fig $1 \mathrm{a}$ and fig $1 \mathrm{~b}$ in online supplement).

The presence of nodular pleural thickening was observed in $14 / 33$ patients (42\%) with malignant effusions and in $0 / 19$ benign cases ( $\chi^{2} 1 \mathrm{df} 11.03, \mathrm{p}=0.009$ ), giving a sensitivity of $42 \%$ (95\% CI $26 \%$ to $61 \%$ ), specificity $100 \%$ (95\% CI $82 \%$ to $100 \%$ ), PPV $100 \%$ and NPV $50 \%$, LR+ve = infinite).

Thickening/nodularity of the visceral pleura was detected on TUS in 5/33 patients with malignant effusions (15\%) and 0/19 benign cases (sensitivity 15\%), and was not seen on CECT in any case.

\section{Other TUS morphological characteristics}

Visceral pleural thickening was observed in five patients (all with malignancy) and occurred in the absence of associated parietal pleural thickening in four cases (see fig 2 in online supplement).

Pleural thickening was hypoechoic relative to the intercostal muscles in all patients with benign pleural thickening $(n=5$; see fig $3 a$ and $3 b$ in online supplement). In malignant pleural thickening, echotexture was non-specific and appeared hypoechoic, hyperechoic or isoechoic.

The morphological characteristics of the diaphragm were compared between malignant and benign groups. Resolution of all five layers of the diaphragm was not possible in 10/33 patients (30\%) with malignant effusions and $1 / 19(5 \%)$ patients with a benign effusion ( $\chi^{2} 1 \mathrm{df} 4.53, \mathrm{p}=0.033$; see fig $4 \mathrm{a}$ in online supplement). Diaphragmatic nodules measuring $2-22 \mathrm{~mm}$ in size
Table 2 Method of final diagnosis

\begin{tabular}{lc}
\hline Diagnostic method & No of patients \\
\hline Malignant disease ( $\mathrm{n}=33$ ) & 14 \\
Pleural histology: & 10 \\
Percutaneous biopsy & 4 \\
Thoracoscopic biopsy & 5 \\
Other histology: & 2 \\
Bronchoscopy & 2 \\
Supraclavicular lymph node biopsy & 1 \\
Axillary lymph node biopsy & 11 \\
Pleural fluid cytology: & 3 \\
Clinical follow-up and repeat radiology & 33 \\
Total & \\
Benign disease* (n=19) & 6 \\
Pleural histology†: & 4 \\
Percutaneous biopsy & 2 \\
Thoracoscopic biopsy & 3 \\
Other histology: & 2 \\
Supraclavicular lymph node biopsy & 1 \\
Pericardectomy & 10 \\
Pleural fluid cytology negative & 19 \\
Total &
\end{tabular}

Pleural fluid cytology was taken as confirmation of malignancy only in the presence of a confident histopathology opinion reporting confirmed malignant cells on cytology \pm immunostaining as required. In these cases, no further investigation to establish diagnosis was required. Negative pleural fluid cytology was not taken as definitive proof of benign aetiology (hence clinical follow-up as below was pursued).

*All patients with a diagnosis of benign disease were followed up for a period of 12 months (at our institution or the referring institution), in which time there was no evidence of malignancy developing within the pleura or elsewhere as the cause of the presenting pleural effusion.

$\uparrow$ Two patients underwent non-diagnostic percutaneous pleural biopsy and subsequent diagnostic thoracoscopy. These patients are only listed once as thoracoscopic biopsies.

were identified in 10/33 patients with a malignant effusion and in none of the patients with a benign effusion (table 3 and fig $4 \mathrm{~b}$ in online supplement). The diaphragm was thickened in 23 patients; this was nodular/irregular in 15 cases (fig $4 \mathrm{c}$ in online supplement) and smooth in 8. Using a TUS threshold value of diaphragmatic thickness of $>7 \mathrm{~mm}$ as suggestive of malignancy, TUS has a sensitivity of $42 \%$ (95\% CI $26 \%$ to $61 \%$ ), specificity of $95 \%(95 \%$ CI $74 \%$ to $99 \%$ ), PPV $93 \%$ and NPV $49 \%$ (LR+ve = 8.4) for differentiating malignant from benign disease.

\section{CT diagnosis}

The median time interval between the CT examinations and the ultrasound examination was 1 day (range 0-41 days). CT correctly differentiated malignant from benign pleural effusion in $49 / 52$ patients (sensitivity $97 \%$ (95\% CI $84 \%$ to $99 \%$ ), specificity $89 \%$ (95\% CI 67\% to 99\%), PPV 94\% and NPV 94\%, $\mathrm{LR}+\mathrm{ve}=8.8$ ). CT identified two patients as false positive (ie, scored malignant on CT, final diagnosis benign). Both of these patients had extensive supraclavicular and mediastinal lymphadenopathy with distortion of the airways and bilateral effusions suggestive of lymphoma. Subsequent ultrasoundguided core biopsy of the supraclavicular lymphadenopathy diagnosed tuberculosis in both cases.

\section{TUS versus CT diagnosis}

Comparison of TUS and CECT diagnosis is made as CECT is considered to be the gold standard investigation for patients with suspected malignant effusion. The CECT true positive rate was $97 \%(32 / 33)$ compared with $79 \%(26 / 33)$ for TUS. Of the seven patients with a false negative diagnosis on TUS, in six the pleural surfaces appeared normal on both US and CT and the 
Table 3 Sonographic findings in patients with malignant and benign pleural effusions

\begin{tabular}{|c|c|c|c|}
\hline Sonographic finding & $\begin{array}{l}\text { Malignant } \\
\text { disease } \\
(\mathrm{n}=33)\end{array}$ & $\begin{array}{l}\text { Benign } \\
\text { disease } \\
(n=19)\end{array}$ & $\begin{array}{l}\text { p value } \\
\text { (Fisher } \\
\text { exact } \chi^{2}\end{array}$ \\
\hline Parietal pleural thickening & 16 & 5 & 0.090 \\
\hline$>1 \mathrm{~cm}$ & 14 & 1 & 0.004 \\
\hline$<1 \mathrm{~cm}$ & 2 & 4 & 0.175 \\
\hline Nodular & 14 & 0 & 0.001 \\
\hline Smooth & 2 & 5 & 0.085 \\
\hline $\begin{array}{l}\text { Visceral pleural thickening/ } \\
\text { nodularity }\end{array}$ & 5 & 0 & 0.145 \\
\hline Diaphragmatic thickening & 19 & 4 & 0.019 \\
\hline Nodular & 15 & 0 & $<0.001$ \\
\hline Smooth & 4 & 4 & 0.443 \\
\hline Diaphragmatic nodules & 10 & 0 & 0.009 \\
\hline \multicolumn{4}{|l|}{ Size of effusion } \\
\hline Small & 9 & 6 & 0.541 \\
\hline Moderate & 17 & 7 & 0.391 \\
\hline Large & 7 & 6 & 0.510 \\
\hline \multicolumn{4}{|l|}{ Nature of effusion } \\
\hline Anechoic/simple & 14 & 11 & 0.389 \\
\hline Anechoic/septated & 14 & 8 & 1.000 \\
\hline Echogenic & 5 & 0 & 0.145 \\
\hline Liver metastases* & 2 & 0 & 0.527 \\
\hline \multirow[t]{3}{*}{ Overall TUS diagnostic rate $\uparrow$} & $26 / 33$ & 19/19 & $\mathrm{N} / \mathrm{A}$ \\
\hline & Sensitivity $79 \%$ & $\begin{array}{l}\text { Specificity } \\
100 \%\end{array}$ & \\
\hline & $N P V=73 \%$ & $P P V=100 \%$ & \\
\hline
\end{tabular}

NPV, negative predictive value; PPV, positive predictive value; TUS, thoracic ultrasound.

*Liver metastases were the sole abnormality detected in one patient on TUS $\dagger$ Diagnostic rate of TUS based on the criteria mentioned in the Methods section (ie, diaphragmatic and parietal pleural nodule or nodules, pleural thickening $>1 \mathrm{~cm}$ or hepatic metastasis).

CT diagnosis of malignancy was based on the presence of intraparenchymal masses, nodal enlargement or pulmonary metastasis. In the remaining patient (false negative on both CECT and TUS) there was a previous history of asbestos exposure, smooth pleural thickening on CT scanning which was not visualised on TUS and subsequent thoracoscopic-guided biopsy showed benign fibrinous thickening. A repeat CT scan performed 3 months later due to increasing chest pain and clinical deterioration showed increased pleural thickening and a clinicoradiological diagnosis of mesothelioma was made. The patient's clinical course was consistent with this diagnosis (death occurred 2 weeks later). Benign disease $(n=19)$ was correctly diagnosed in all cases using TUS and in 89\% (17/19) using CECT (tuberculosis misdiagnosed as malignancy in two cases as above).

\section{DISCUSSION}

This is the first analysis to specifically assess the usefulness of TUS in the diagnosis of suspected malignant pleural effusion. The "definitive" diagnosis used in this patient population was well defined and included clinical follow-up data of 12 months for all patients with presumed benign disease. Hitherto, TUS has been used as a technique to confirm the presence of fluid, assess fluid characteristics (eg, septated/echogenic) or to guide intervention. Using similar morphological criteria as those used in CECT, ${ }^{12}$ our results show that TUS is able to distinguish malignant from benign effusions with an overall sensitivity of $79 \%$ and specificity of $100 \%$ (table 3). These figures are comparable to the previously published data on diagnosis of
Table 4 Sensitivities and specificities for ultrasound and CTdetermined criteria that are suggestive of malignant pleural disease.

\begin{tabular}{|c|c|c|c|c|}
\hline & \multirow{2}{*}{\multicolumn{2}{|c|}{$\begin{array}{l}\text { This study } \\
\text { (n= 52) } \\
\text { Ultrasound }\end{array}$}} & \multirow{2}{*}{\multicolumn{2}{|c|}{$\begin{array}{l}\begin{array}{l}\text { Leung et } a f^{12} \\
(\mathrm{n}=74)\end{array} \\
\text { CT scanning }\end{array}$}} \\
\hline & & & & \\
\hline & $\begin{array}{l}\text { Sensitivity } \\
(\%)\end{array}$ & $\begin{array}{l}\text { Specificity } \\
(\%)\end{array}$ & $\begin{array}{l}\text { Sensitivity } \\
(\%)\end{array}$ & $\begin{array}{l}\text { Specificity } \\
(\%)\end{array}$ \\
\hline $\begin{array}{l}\text { Parietal pleural } \\
\text { thickening }>1 \mathrm{~cm}\end{array}$ & 42 & 95 & 56 & 88 \\
\hline $\begin{array}{l}\text { Nodular pleural } \\
\text { thickening }\end{array}$ & 42 & 100 & 36 & 85 \\
\hline $\begin{array}{l}\text { Visceral pleural } \\
\text { thickening }\end{array}$ & 15 & 100 & NA & NA \\
\hline $\begin{array}{l}\text { Diaphragmatic thickening } \\
>7 \mathrm{~mm}\end{array}$ & 42 & 95 & NA & NA \\
\hline $\begin{array}{l}\text { Diaphragmatic layers } \\
\text { resolved }\end{array}$ & 30 & 95 & NA & NA \\
\hline Diaphragmatic nodules & 30 & 100 & NA & NA \\
\hline Overall & 79 & 100 & 72 & 83 \\
\hline
\end{tabular}

$\mathrm{NA}$, not assessed.

malignant effusion with CECT alone (table 4), although this was not the primary aim of the study. TUS is a quick, relatively inexpensive and harmless procedure which is increasingly being performed by chest physicians; if these results are confirmed in larger studies, TUS may become a valuable adjunct in the diagnosis of malignant pleural effusion.

Beyond the criteria defined in previous CT studies, we have found several TUS morphological features apparently associated with malignant pleural effusion. In the absence of parietal pleural thickening, visceral thickening and visceral nodularity were associated with malignancy and not apparently visible on CECT. Necropsy reports have shown that the parietal pleura may be less frequently involved with metastatic disease than the visceral pleura. ${ }^{19}$ This suggested pathogenesis would be in keeping with our findings in which $4 / 5$ patients demonstrated visceral pleural thickening or nodularity suggestive of visceral deposits in the absence of associated parietal pleural thickening.

Diaphragmatic abnormalities have been shown in this study to predict malignant disease. The normal diaphragm is usually well defined on TUS (due to the presence of fluid in the costophrenic recess), $<5 \mathrm{~mm}$ thick and divided into five distinct layers sonographically. Inability to resolve diaphragmatic layers, the presence of diaphragmatic nodules and diaphragm thickness of $>7 \mathrm{~mm}$ were associated with malignant pleural disease in this study. TUS appears to be more sensitive than CECT in demonstrating visceral pleural disease and diaphragmatic nodularity.

Although previous studies suggest that differentiating malignant parietal pleural thickening and chronic pleural fibrosis can be difficult on TUS, ${ }^{20}$ our results suggest that TUS is relatively accurate. In our series, six patients were diagnosed with chronic pleural fibrosis following percutaneous or thoracoscopic biopsy and TUS diagnosed all six cases as benign. In contrast to this, the CT appearances were highly suspicious of malignancy in one patient, indeterminate in one and benign in four cases.

Of the seven false negative cases on TUS, six (86\%) had normal pleural surfaces when assessed by CECT. The absence of detectable pleural disease on thoracic CT in the presence of a malignant pleural effusion has been previously reported. ${ }^{21}$ In this case, CECT has the clear advantage over TUS of being able to assess for the presence of disease elsewhere (eg, lung parenchyma, mediastinal nodes, distant metastases), although TUS may be used to assess for the presence of liver metastasis, which was the only abnormality found in one patient. 
There are several limitations to this study. Initial TUS examinations were performed by a single observer with 5 years experience in ultrasound but only 6 months experience in pleural US. Although the results might have been improved if a more experienced operator had performed the scans, the close agreement achieved between observers is reassuring. Second, the mediastinal surfaces were not scanned in this study. Although technically challenging, this is possible in patients with a large pleural effusion and may have improved the diagnostic accuracy. Third, this study comprised a relatively small number of patients $(n=52)$ recruited from a tertiary referral centre for pleural disease. The incidence of malignancy in this population is likely to be higher than that seen in a less specialist centre, and many of these patients had already undergone prior investigations with negative results (most commonly, cytology-negative pleural aspirate). However, there is no reason to believe that the morphological characteristics on TUS here shown to be associated with malignant pleural disease should be any different. The relatively high incidence of mesothelioma in this series is likely to be attributable to the institution being the local tertiary referral centre for pleural disease. This may have resulted in a biased estimate of the diagnostic performance of TUS if mesothelioma is more easily detected at ultrasound. Finally, although we have compared the specificity and sensitivity of TUS with CECT, this study is underpowered to conduct a non-inferiority analysis of the two techniques.

What implications does this study have for clinical practice? CECT has a high sensitivity and specificity for malignant pleural effusion and is the usual next radiological investigation after the chest radiograph. The sensitivity of CECT in published studies is high, although the specificity for TUS in this study is higher than that reported for CECT (table 4). However, there are often delays in obtaining CT scans and TUS is easier to access and is increasingly being performed by chest physicians. The reasonable specificity and high PPV demonstrated using TUS in this study suggest that TUS may be useful as an initial test (for example, in the outpatient setting) in the investigation of patients with pleural effusion of unknown aetiology, which may streamline the diagnostic process. Larger studies in populations with a lower pretest probability of malignant pleural disease are required to assess this fully, including studies to determine whether TUS conducted by non-radiologists has a similar predictive value.

In conclusion, blinded assessment of TUS has shown that the technique has a reasonable sensitivity and high specificity and PPV for malignant pleural effusion in the absence of empyema. The presence of pleural thickening $>1 \mathrm{~cm}$, diaphragmatic nodularity or thickening $>7 \mathrm{~mm}$, visceral pleural thickening and pleural nodularity/irregularity are associated with malignancy. TUS may therefore be useful, not only in guiding thoracocentesis and biopsy, but also as an adjunct to aid diagnosis. Further studies are required to confirm this finding.

Funding: None specific to this study. NMR is funded by an MRC Training Fellowship. Competing interests: None.

Ethics approval: The study was discussed with the Chair of the Oxford Research Ethics Committee and considered an audit of practice not requiring specific ethics approval.

\section{REFERENCES}

1. Maskell NA, Butland RJ. BTS guidelines for the investigation of a unilateral pleural effusion in adults. Thorax 2003;58(Suppl 2):ii8-17.

2. American Thoracic Society. Management of malignant pleural effusions. Am J Respir Crit Care Med 2000;162:1987-2001.

3. Antony VB, Loddenkemper R, Astoul P, et al. Management of malignant pleural effusions. Eur Respir J 2001;18:402-19.

4. Rahman NM, Davies RJ, Gleeson FV. Investigating suspected malignant pleural effusion. BMJ 2007;334:206-7.

5. Dresler CM. Systemic distribution of talc. Chest 1999;116:266.

6. Hirsch A, Ruffie P, Nebut M, et al. Pleural effusion: laboratory tests in 300 cases. Thorax 1979;34:106-12.

7. Prakash UB, Reiman HM. Comparison of needle biopsy with cytologic analysis for the evaluation of pleural effusion: analysis of 414 cases. Mayo Clin Proc 1985;60:158-64.

8. Salyer WR, Eggleston JC, Erozan YS. Efficacy of pleural needle biopsy and pleural fluid cytopathology in the diagnosis of malignant neoplasm involving the pleura. Chest 1975;67:536-9.

9. Renshaw AA, Dean BR, Antman KH, et al. The role of cytologic evaluation of pleural fluid in the diagnosis of malignant mesothelioma. Chest 1997;111:106-9.

10. Antunes G, Neville E, Duffy J, et al. BTS guidelines for the management of malignant pleural effusions. Thorax 2003;58(Suppl 2):ii29-38.

11. Traill ZC, Davies RJ, Gleeson FV. Thoracic computed tomography in patients with suspected malignant pleural effusions. Clin Radiol 2001;56:193-6.

12. Leung AN, Muller NL, Miller RR. CT in differential diagnosis of diffuse pleural disease. AJR Am J Roentgenol 1990;154:487-92.

13. Scott EM, Marshall TJ, Flower CD, et al. Diffuse pleural thickening: percutaneous CTguided cutting needle biopsy. Radiology 1995;194:867-70.

14. Qureshi NR, Gleeson FV. Imaging of pleural disease. Clin Chest Med 2006;27:193-213

15. Royal College of Radiology. Ultrasound training recommendations for medical and surgical specialties. 2006. http://www.rcr.ac.uk/docs/radiology/pdf/ultrasound.pdf.

16. Gorg C, Restrepo I, Schwerk WB. Sonography of malignant pleural effusion. Eur Radiol 1997; 7:1195-8.

17. Yang PC, Luh KT, Chang DB, et al. Value of sonography in determining the nature of pleural effusion: analysis of 320 cases. AJR Am J Roentgenol 1992;159:29-33.

18. Venekamp LN, Velkeniers B, Noppen M. Does 'idiopathic pleuritis' exist? Natural history of non-specific pleuritis diagnosed after thoracoscopy. Respiration 2005; 72:74-8.

19. Sahn SA. Malignant pleural effusions. Semin Respir Crit Care Med 2001;22:607-16

20. Tsai TH, Yang PC. Ultrasound in the diagnosis and management of pleural disease. Curr Opin Pulm Med 2003;9:282-90.

21. Waite RJ, Carbonneau RJ, Balikian JP, et al. Parietal pleural changes in empyema: appearances at CT. Radiology 1990;175:145-50. 